\title{
Terremoto en Chile: estrés agudo y estrés post-traumático en mujeres en tratamiento por depresión grave
}

\author{
VERÓNICA VITRIOL G. ${ }^{1}$, ALFREDO CANCINO A. ${ }^{2}$, \\ PAULA RIQUELME S. ${ }^{\mathrm{a}}$ IGNACIA REYES F. ${ }^{\mathrm{a}}$
}

${ }^{1}$ Escuela de Medicina, Universidad de TalcaUnidad de Salud Mental, Hospital base de Curicó 2Unidad de Salud Mental, Cesfam Curicó-centro, Curicó. aPsicóloga.

Este trabajo no contó con financiamiento y los autores declaran no tener conflictos de interés.

Recibido el 21 de julio de 2012, aceptado el 30 de octubre de 2012

Correspondencia a: Verónica Vitriol G. Teléfono: 075-317471 E-mail: vvitriol@utalca.cl, verovitriol@gmail.com

\section{Earthquake in Chile: acute stress and post traumatic stress disorder among women in treatment for severe depression}

Background: Depression is a risk factor for developing secondary post-traumatic stress disorder. The earthquake on February 27th, 2010, destroyed the Hospital of Curicó. Despite the prevailing chaos, patients in treatment for severe depression actively maintained their treatment from the first post-earthquake working day. Aim: To determine prevalence of acute stress (AS) and post-traumatic stress disorder (PTSD) after the earthquake among women in treatment for severe depression. Material and Methods: Seventy five women aged 20 to 73 years in treatment for depression for at least six months before the occurrence of the earthquake, who continued in follow up after the event, were studied. According to the Tenth International Classification of Diseases, the frequency of AS was assessed during the consultation occurring one month after the event and the frequency of PTSD was assessed during the consultation occurring six months after the event. Results: Fifty nine percent of women had AS symptoms at the first month and $53.3 \%$ had PTSD, six months later. AS was significantly associated with a low educational level. PTSD was significantly associated with prior occurrence of AS and a history of childhood trauma history. Conclusions: A high frequency of PTSD was observed in this group of depressive patients, which was significantly associated with a history of previous AS and childhood trauma.

(Rev Med Chile 2013; 141: 338-344).

Key words: Depression; Earthquakes; Hospitals, public.
1 127 de febrero de 2010, un megaterremoto de 8,8 grados Richter, seguido de un tsuna$\mathrm{mi}$, afectó un vasto territorio de la zona central de Chile ${ }^{1}$, generando daños transversales en economía, vivienda, conectividad, infraestructura hospitalaria, vidas humanas y deterioro psicológico ${ }^{2}$.

Estudios realizados en los últimos 30 años estiman que entre 30 y $50 \%$ de la población general expuesta a un desastre natural sufre alguna perturbación psicológica en los meses siguientes al evento ${ }^{3,4}$. Ésta se puede manifestar bajo la forma de cuadros transitorios como distress subclínico, estrés agudo (EA) y trastornos adaptativos, hasta patologías de mayor duración como trastorno de estrés postraumático (TEPT), trastornos depresivos, cuadros ansiosos, abuso de alcohol/drogas, y trastornos somatomorfos ${ }^{5-7}$.

El TEPT es la principal patología que se presenta en la población general después de un desastre 
natural, con prevalencias que fluctúan entre 5 y $23 \%$, seguida por los trastornos depresivos, con prevalencias entre 12 y $16 \% \%^{8,9}$. Los síntomas de TEPT se han asociado a las consecuencias directas del evento propiamente tal ${ }^{10}$. En cambio, las alteraciones anímicas se han relacionado en forma más específica con vulnerabilidades personales como bajo grado de educación, bajo nivel socioeconómico y estresores enfrentados a lo largo del ciclo vital $^{10}$. Por otro lado, se ha evidenciado que los síntomas de TEPT tenderían a disminuir en el tiempo y no así los síntomas depresivos ${ }^{11}$, y que la coexistencia de síntomas depresivos con los de TEPT empeora la evolución de este último ${ }^{12}$.

La mayoría de los estudios sobre prevalencia de TEPT vinculado a desastres naturales se ha realizado en población general ${ }^{8}$ y han establecido que el antecedente de patología psiquiátrica constituye uno de los factores de riesgo para su desarrollo ${ }^{7,8,13}$.

Investigar la prevalencia de TEPT postdesastre natural, específicamente en sujetos que cursan cuadros depresivos, adquiere relevancia considerando que los estudios epidemiológicos realizados en situaciones habituales demuestran la alta comorbilidad entre depresión y TEPT ${ }^{14-16}$, lo que además está asociado a una mayor gravedad clínica y a un mayor gasto de recursos en salud $^{17-19}$. Ambas patologías comparten factores de riesgo como el sexo femenino y los antecedentes traumáticos infantiles ${ }^{20,21}$, y cada una es factor de vulnerabilidad neurobiológica para el desarrollo de la otra ${ }^{22}$.

En Chile, la depresión es un importante problema de salud pública ${ }^{23}$. Desde el año 2006, está incluida entre las patologías con garantías explícitas en salud, contándose con sucesivas Guías Clínicas ministeriales estructuradas para su tratamiento ${ }^{24-26}$. De hecho, al momento del terremoto, existía un grupo importante de pacientes que estaba recibiendo tratamiento por depresión en distintos niveles de la red asistencial.

Curicó, ciudad ubicada $180 \mathrm{~km}$ al sur de la capital, muy cerca del epicentro, resultó extensamente devastada por este desastre natural. El área central resultó gravemente destruida, sin luz, comunicaciones ni servicios básicos en un lapso de 5 a 15 días. Su hospital colapsó, al igual que los demás centros asistenciales de la zona ${ }^{27}$. La unidad de salud mental fue desalojada de sus dependencias y la atención de los pacientes se re-estructuró desde el primer día hábil posterior al terremoto en tres lugares sucesivos, con la concurrencia tanto de profesionales como de usuarios.

Dentro de la población que asistió precozmente a sus controles habituales destacó el grupo de pacientes que se encontraba en tratamiento por depresión grave. Específicamente en esta población, el equipo profesional local venía investigando, desde el año 2002, las consecuencias clínicas asociadas a la alta frecuencia de antecedentes traumáticos tempranos, que confieren mayor gravedad y cronicidad a la patología depresiva ${ }^{28-31}$.

Considerando que Chile es un país altamente sísmico, la importancia de la depresión en nuestro medio, su relación con el TEPT y la falta de estudios que den cuenta de las consecuencias de los desastres naturales en grupos clínicos específicos, se desarrolló la presente investigación con el objetivo de determinar las prevalencias de estrés agudo (EA) y estrés postraumático (TEPT), y su relación con antecedentes demográficos, clínicos y con las consecuencias del terremoto del 27 de febrero de 2010, en una muestra de mujeres que se encontraba en tratamiento por depresión grave en el Hospital de Curicó.

\section{Material y Método}

El diseño de esta investigación corresponde a un estudio descriptivo de prevalencias y asociación simple.

La muestra fue conformada por mujeres mayores de 15 años con diagnóstico de depresión grave de acuerdo a la CIE-10 y a las Guías Clínicas ministeriales ${ }^{24-26,32}$, que se encontraban en tratamiento a lo menos durante seis meses antes del terremoto en la Unidad de Salud Mental del Hospital de Curicó y que durante el primer mes post-desastre asistieron al control habitual con su psiquiatra tratante, profesional con años de desempeño en dicha unidad.

Contando con las correspondientes autorizaciones y con la firma de un consentimiento informado, se constituyó una muestra de 75 mujeres que fueron evaluadas durante el primer mes y re-evaluadas a los seis meses después del terremoto. Así, de acuerdo a la CIE $10^{30}$, en el control del primer mes, la psiquiatra tratante determinó clínicamente la presencia de EA y en el control de los seis meses se verificó clínicamente la presencia de TEPT.

A partir de los registros clínicos ya existentes se obtuvo la siguiente información: 
- Datos socio-demográficos: edad, estado civil, escolaridad y ocupación al momento del estudio.

- Antecedentes relevantes asociados directamente al terremoto: haber vivenciado el maremoto, haber sufrido pérdidas materiales, daños físicos o la muerte de algún familiar.

- Información clínica sobre el diagnóstico de depresión, tiempo de tratamiento en la unidad, historia de trauma infantil y la ocurrencia alguna vez en la vida de intento de suicidio y de hospitalización debida a cuadro depresivo.

Para los fines de este estudio, se consideró 'historia de trauma infantil' el reporte de tres o más de los siguientes antecedentes acaecidos antes de los 15 años de edad: haber vivido abandono por parte del cuidador, haber convivido con familiar que abusara de alcohol/drogas, haber sufrido castigo físico sistemático, haber quedado con algún estigma físico producto de castigo, haber sido testigo de violencia física interpersonal $\mathrm{y} / \mathrm{o}$ haber sido víctima de abuso sexual. Los autores han utilizado esta misma metodología en estudios previos ${ }^{29}$. Este antecedente se encontraba ya registrado en la ficha clínica utilizando una nomenclatura cifrada en clave.

Entre las evaluaciones del primer y sexto mes, las pacientes continuaron su intervención habitual para tratar la depresión, contemplándose tratamiento farmacológico antidepresivo acorde a la Guía Clínica ${ }^{32}$, control psiquiátrico mensual y sesiones de apoyo psicoterapéutico interpersonal una vez a la semana durante el primer mes y en adelante cada 15 días o mensualmente, de acuerdo a la evolución de la paciente.
Los datos fueron procesados utilizando el paquete estadístico SPSS-12, determinando prevalencias de EA y TEPT y las asociaciones simples entre estos diagnósticos y variables demográficas, clínicas y secundarias al terremoto.

Cabe señalar que este trabajo se enmarca en las recomendaciones éticas impartidas por la Sociedad Japonesa de Psiquiatría y Neurología respecto de la investigación en situaciones de desastre ${ }^{33}$.

\section{Resultados}

El promedio de edad de la población estudiada fue de 47 años. Cuarenta y siete por ciento de las pacientes tenía pareja estable, $16 \%$ estaba soltera y $25,6 \%$ separada. Cuarenta por ciento de la muestra contaba con escolaridad inferior a 6 años. Treinta y cinco por ciento desarrollaba alguna ocupación al momento de ocurrido el desastre. Las pacientes habían estado en tratamiento en la unidad durante un promedio de 10,5 años.

Dentro del espectro depresivo, el diagnóstico más prevalente era trastorno depresivo recurrente, que alcanzaba $65,3 \%$ de la muestra. El 65,3\% registraba el antecedente de al menos un intento de suicidio, 52\% había requerido de hospitalización y $71 \%$ tenía antecedentes de trauma infantil.

Respecto a las consecuencias vinculadas al terremoto, sólo una paciente vivenció el tsuna$\mathrm{mi}, 37,3 \%$ de la muestra experimentó pérdidas materiales importantes y $4 \%$ enfrentó la pérdida de familiares cercanos. Ninguna de las pacientes sufrió daño a la integridad física (Tabla 1).

Luego del evento, $58,7 \%(n=44)$ de la muestra presentó manifestaciones clínicas de EA asociado

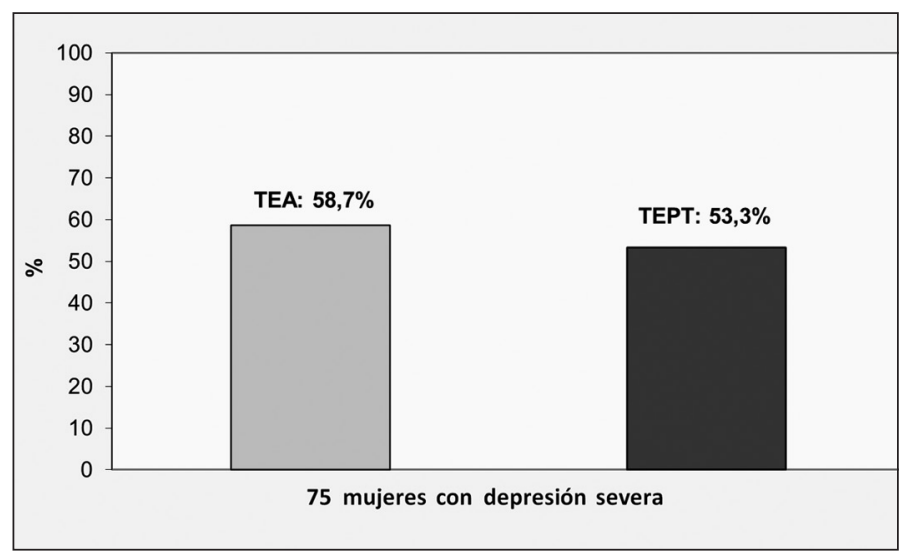

Figura 1. Prevalencias de estrés agudo al primer mes y de trastorno de estrés postraumático al sexto mes post-terremoto 27-febrero-2010, en 75 mujeres con depresión severa. Unidad Salud Mental, Curicó (2010). 
Tabla 1. Antecedentes socio-demográficos, clínicos y asociados al impacto del terremoto 27-F, en 75 mujeres con depresión severa, Unidad Salud Mental, Curicó (2010)

\begin{tabular}{|c|c|c|}
\hline & $\mathbf{n}$ & $\%$ \\
\hline \multicolumn{3}{|l|}{$\begin{array}{l}\text { Antecedentes } \\
\text { socio-demográficos }\end{array}$} \\
\hline Edad promedio & $\begin{array}{l}47 \text { años } \\
(20-73)\end{array}$ & \\
\hline \multicolumn{3}{|l|}{ Escolaridad } \\
\hline - Sin información & 5 & 6,7 \\
\hline - Básica & 30 & 40 \\
\hline - Media & 27 & 36 \\
\hline - Técnico-profesional & 9 & 12 \\
\hline - Superior & 4 & 5,3 \\
\hline \multicolumn{3}{|l|}{ Estado civil } \\
\hline - Sin información & 2 & 2,7 \\
\hline - Soltera & 12 & 16 \\
\hline - Casada & 34 & 45,3 \\
\hline - Conviviente & 3 & 4 \\
\hline - Separada & 20 & 26,7 \\
\hline - Viuda & 4 & 5,3 \\
\hline \multicolumn{3}{|l|}{ Ocupación } \\
\hline & 26 & 37 \\
\hline - No & 49 & 63 \\
\hline \multicolumn{3}{|l|}{$\begin{array}{l}\text { Antecedentes clínicos al } \\
\text { momento del terremoto }\end{array}$} \\
\hline \multicolumn{3}{|l|}{ Tipo de depresión } \\
\hline - Trastorno depresivo recurrente & 49 & 65,3 \\
\hline - Trastorno bipolar & 13 & 17,3 \\
\hline - Depresión refractaria & 6 & 8 \\
\hline $\begin{array}{l}\text { - Trastorno depresivo sin } \\
\text { especificación }\end{array}$ & 7 & 9,4 \\
\hline Intento de suicidio previo & 49 & 65,3 \\
\hline Hospitalización previa & 39 & 52 \\
\hline Historia de trauma infantil & 53 & 70,7 \\
\hline Tiempo promedio tratamiento & 10,5 años & \\
\hline \multicolumn{3}{|l|}{$\begin{array}{l}\text { Antecedentes vinculados a las } \\
\text { consecuencias del terremoto }\end{array}$} \\
\hline Pérdidas materiales & 28 & 37,3 \\
\hline Pérdidas humanas & 3 & 4 \\
\hline
\end{tabular}

Tabla 2. Asociaciones entre EA y TEPT, y entre trauma infantil y TEPT, en 75 mujeres con depresión severa. Unidad Salud Mental, Curicó (2010)

\begin{tabular}{|lccccc|}
\hline & n & TEPT & \multicolumn{2}{c|}{ No TEPT } \\
\hline TEA & 33 & 82,5 & 11 & $31,4^{*}$ \\
No TEA & 7 & 17,5 & 24 & 68,6 \\
Trauma & 35 & 87,8 & 18 & $51,4^{* *}$ \\
No trauma & 5 & 12,2 & 17 & 48,6 \\
\hline${ }^{*} \chi^{2}=20,07$. & OR $=10,28(3,4-30,4)$ & $p<$ & $<, 001$. \\
${ }^{*} \chi^{2}=11,93$. OR $=6,6$ & $(2,09-20,98)$ & $p<0,003$.
\end{tabular}

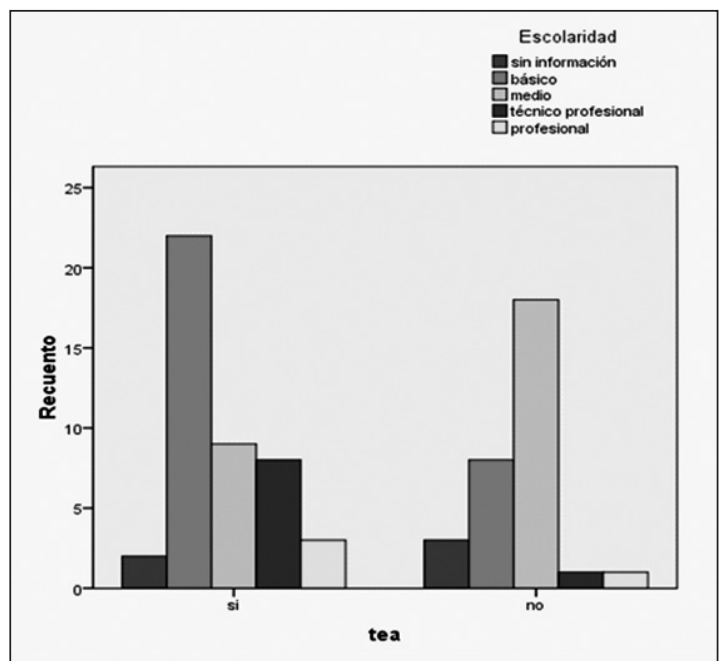

Figura 2. Distribución de escolaridad de acuerdo a presencia o no de EA, post-terremoto 27-febrero-2010 en 75 mujeres con depresión severa. Unidad Salud Mental, Curicó (2010). $\chi^{2}=14,4 ; g l$; $p<0,006$

al terremoto y al cabo de seis meses 53,3\% $(\mathrm{n}=40)$ desarrolló TEPT en relación al terremoto (Figura 1).

Se estableció una relación significativa entre prevalencia de EA y bajo nivel de escolaridad $\left(\chi^{2}=14,33 ; \mathrm{p}<0,006\right)$ (Figura 2). Asimismo, el TEPT se asoció significativamente con la ocurrencia previa de EA $\left(\chi^{2}=20,78 ; \mathrm{p}<0,001\right.$; $\mathrm{OR}=10,28)$ y con antecedentes de trauma infantil $\left(\chi^{2}=11,934 ; \mathrm{p}<0,003 ; \mathrm{OR}=6,6\right)($ Tabla 2).

\section{Discusión}

Esta investigación fue factible gracias a una coyuntura local que excepcionalmente posibilitó el precoz restablecimiento del contacto entre la instancia asistencial y una población de alta vulnerabilidad psicológica frente a las situaciones traumáticas. Lo anterior se refiere a que, aun en medio del caos imperante, el acceso al servicio de salud mental no resultó interrumpido, se realizó una rápida reorganización de la atención clínica por parte del equipo de salud, se garantizó el suministro de los medicamentos y las pacientes lograron acudir a sus citaciones a pesar de tres sucesivos traslados de la Unidad de Salud Mental. Es decir, se logró cumplir con las recomendaciones mínimas impartidas por los manuales vigentes 
respecto al manejo de pacientes con trastornos mentales en situaciones de emergencia ${ }^{33-35}$.

Se pudo evidenciar que la debida implementación de las Guías Ministeriales de salud mental difundidas en las últimas décadas ha favorecido la disponibilidad de orientaciones técnicas y recursos necesarios para facilitar la continuidad terapéutica en pacientes graves, aún bajo circunstancias de catástrofe $^{24,32}$. Acorde a lo anterior, se enfatiza la centralidad del vínculo terapéutico generado en el trabajo habitual con una población bajo control de alta vulnerabilidad. En este sentido, se pudo constatar cómo las propias pacientes buscaron activamente mantener el contacto con los profesionales tratantes.

La prevalencia de EA en relación al terremoto que presentó esta población es concordante con la encontrada en estudios de población general sometidas a situaciones de catástrofe y que fluctúa entre 7 y $68 \%{ }^{6,36,37}$. Sin embargo, la prevalencia de TEPT post-terremoto resultó ser considerablemente más alta que 21,4\% informado por el Ministerio de Planificación en la población general de la región del Maule a los tres meses del desastre ${ }^{2}$. Esta mayor prevalencia puede explicarse en base a que nuestra muestra de estudio presenta al menos dos de los factores de riesgo descritos para el desarrollo de TEPT: el género femenino y la presencia de psicopatología previa ${ }^{13}$. Otro antecedente que podría explicar en parte esta mayor prevalencia de TEPT es el hallazgo encontrado en el año 2006, en la misma Unidad de Salud Mental de Curicó, en una muestra de mujeres que ingresaba a tratamiento por depresión severa, en quienes, mediante la aplicación del CIDI, se constató una prevalencia de vida de TEPT por algún trauma de $49,2 \%{ }^{39}$. En dicho estudio, el trauma más frecuentemente recordado fue el haber visto a alguien herido de gravedad y en quinto lugar el haber vivido un desastre natural.

A diferencia de los estudios en población general que señalan que la presencia de EA y TEPT post-catástrofe se relaciona mayormente con las consecuencias propias y directas del desastre ${ }^{7,13}$, en esta muestra clínica los antecedentes socioculturales y de vulnerabilidad personal fueron respectivamente los que se asociaron al desarrollo de EA (baja escolaridad) y TEPT (antecedente de trauma infantil). Lo anterior se podría explicar considerando que nuestro grupo de estudio estaba íntegramente conformado por personas portadoras de depresión severa, que además no sufrieron el impacto directo del tsunami, ni desplazamiento territorial, ni daño físico personal y de las cuales sólo una paciente vivenció la muerte de un familiar.

Por otra parte, una vez más se evidencia la relación altamente significativa entre la presencia de EA y el desarrollo ulterior de TEPT ${ }^{37,38}$ y la relación del antecedente de trauma infantil con el desarrollo de TEPT ${ }^{13}$ Sin embargo, resulta interesante que en nuestra investigación el trauma infantil no se asoció significativamente con la presencia de EA. Este hallazgo es concordante con lo encontrado en un estudio realizado en la población afectada por el huracán Katrina en la cual tampoco se logró correlacionar la historia traumática previa con la ocurrencia de EA post-desastre ${ }^{7}$. Los autores de este estudio postularon que la falta de relación entre los antecedentes traumáticos biográficos y el EA se debería a que el impacto de la emergencia inmediata habría minimizado la relevancia que la historia traumática previa hubiese tenido para los afectados. Sin embargo, cabe señalar que en dicho estudio estos antecedentes fueron recolectados inmediatamente después de la emergencia, a diferencia de nuestra investigación, en la cual la historia traumática infantil constituía un dato ya consignado antes del desastre natural e independiente de la contingencia ${ }^{27,28}$. Este hallazgo, que requiere aún mayor investigación, pudiese ser explicado considerando que el EA constituye una respuesta inespecífica a experiencias extremas que evolucionaría hacia la resolución dentro del primer mes, lo que -expresado metafóricamente- se podría definir como una herida que logra cicatrizar. En cambio, las vulnerabilidades biológicas y psicológicas inespecíficas remanentes del trauma temprano en pacientes expuestos a nuevos traumas impedirían que la herida originada en relación al trauma actual cicatrice totalmente o facilitarían que heridas antiguas pudieran ser re-abiertas las que operarían como punto de inicio para el desarrollo del TEPT asociado al trauma del presente.

Los hallazgos encontrados en cuanto a la prevalencia de TEPT en pacientes con depresión y su asociación con experiencias traumáticas previas son concordantes con evidencias recientes y resaltan la importancia de investigar y capacitar a los equipos sobre el abordaje y tratamiento de dichas situaciones traumáticas. El tratamiento realizado para la depresión en las condiciones clínicas 
regulares, parece ser insuficiente para prevenir el desarrollo de TEPT post- desastre natural. Estos resultados sugieren que en pacientes consultantes por depresión se requeriría investigar la efectividad de intervenciones específicas focalizadas en el trauma e implementadas inmediatamente después de un desastre natural ${ }^{40}$.

Adicionalmente, para conocer resultados más categóricos, sería necesario re-evaluar a estas pacientes tras un año o más de ocurrido el evento.

Como limitaciones, los autores asumen la presencia de algunos sesgos inherentes a una investigación realizada en situación de catástrofe, ya que sólo se estudió a aquellas pacientes que estando previamente en tratamiento lograron proseguir sus controles inmediatamente después del desastre, hubo variabilidad en el momento de evaluación para el diagnóstico de EA dependiente de la fecha de citación (algunas mujeres fueron evaluadas a las $48 \mathrm{~h}$ de sucedido el evento y otras luego de 30 días) y no se evaluó otras variables cuya vinculación al desarrollo del TEPT se conoce por su relevancia, como el grado de apoyo social $\mathrm{y}$ algunos perfiles caracterológicos.

Sin desmedro de lo anterior, a juicio de los autores, esta investigación reviste la ventaja de haber sido desarrollada en un servicio público, en las condiciones naturales del mundo real, pero con ocasión de un mega-evento catastrófico inesperado que, pese a su magnitud, no logró interrumpir el encuentro inmediato entre los profesionales y sus pacientes.

Agradecimientos especiales a la Srta. Marlene Valdés Fuentes, por su valioso aporte en el trabajo de traducción.

\section{Referencias}

1. Vigny C, Socquet A, Peyrat S, Ruegg JC, Métois M, Madariaga R, et al. The $2010 \mathrm{Mw} 8.8$ Maule megathrust earthquake of Central Chile, monitored by GPS. Science 2011; 332 (6036): 1417-21.

2. Ministerio de Planificación Gobierno de Chile: Encuesta Posterremoto. Efectos en la calidad de vida de la población afectada por terremoto/ tsunami, Larrañaga O, Herrera R. 2010.

3. Udomratn P. Mental health and psychosocial consequences of natural disasters in Asia. International Review of Psychiatry 2008; 20 (5): 441-4.

4. Cova F, Rincón P. El terremoto y Tsunami del 27-F y sus efectos en la Salud Mental. Terapia Psicológica 2010; 28 (2): 179-85.

5. Frank H, Hung- Chi W, Pesus Ch, Chao-Yeu S, Kuan-Yi $\mathrm{T}$, Shin-Shin Ch, Ming-Chao Ch, et al. Epidemiologic psychiatric studies on post-disaster impact among ChiChi earthquake survivors in Yu-Chi, Taiwan. Psychiatry Clinical Neurosciences 2007; 61:370-8.

6. Hussain A, Weisaeth L, Heir T. Psychiatric disorders and functional impairment among disaster victims after exposure to a natural disaster: a population based study. J Affect Disord 2011; 128 (2): 135-41.

7. Mills MA, Edmondson D, Park CL. Trauma and stress response among Hurricane Katrina evacuees. Am J Public Health 2007; 97 (1): 116-23.

8. Galea S, Nandi A, Vlahov D. The epidemiology of posttraumatic stress disorder after disasters. Epidemiol Rev 2005; 2778-91.

9. Neria Y, Nandi A, Galea S. Post-traumatic stress disorder following disasters: a systematic review. Psychol Med 2008; 38 (4): 467-80.

10. Tracy M, Norris FH, Galea S. Differences in the determinants of post-traumatic stress disorder and depression after a mass traumatic event. Depress Anxiety 2011; 28 (8): 666-75.

11. Meewisse ML, Olff M, Kleber R, Kitchiner NJ, Gersons BP. The course of mental health disorders after a disaster: predictors and comorbidity. J Trauma Stress 2011; 24 (4): 405-13.

12. Tural U, Onder E, Aker T. Effect of depression on recovery from PTSD. Community Ment Health J 2012; 2 : 161-6.

13. Brewin C, Andrews B, Valentine J. Meta-analysis of risk factors for posttraumatic stress disorder in trauma exposed adults. J Consult Clin Psychol 2000; 68 (5): 748-66.

14. Kessler R, Sonnega A, Bromet E, Hughes M, Nelson C. Post-traumatic stress disorder in the national community survey. Arch Gen Psychiatry 1995; 52: 1048-60.

15. Brady KT, Kileen KT, Brewerton T, Lucerini S. Comorbidity of psychiatric disorders and posttraumatic stress disorder. J Clin Psychiatry 2000; 61 (7): 22-32.

16. Zlotnick C, Johnson J, Kohn R, Vicente B, Risoseco $\mathrm{P}$, Saldivia S. Epidemiology of trauma, post-traumatic stress disorder (PTSD) and co-morbid disorders in Chile. Psychol Med 2006; 36: 1522-33.

17. Chan D, Cheadle AD, Reiber G, Unutzer J, Chaney EF. Health care utilization and its costs for depressed with and without comorbid PTSD symptoms. Psychiatr Serv 2009; 16: 1612-7.

18. Holtzheimer P, Russo J, Zatzick D, Bundy C, Roy-Byrne $P$. The impact of comorbid of post-traumatic stress disorder on short- term clinical outcome in hospitalized 
Terremoto 2010: Estrés agudo y post-traumático en mujeres con depresión - V. Vitriol et al

patients with depression. Am J Psychiatry 2005; 162 (5): 970-6.

19. Green B, Krupnik J, Chung J, Siddique J, Krause E, Revicki D, et al. Impact of PTSD symptoms in a one year outcomes depression trial. J Clin Psychol 2006; 62: 815- 35.

20. Marty C, Carvajal C. Maltrato infantil como factor de riesgo de trastorno por estrés postraumático en la adultez. Rev Chil Neuro-Psiquiat 2005; 43: 180-7.

21. Kendler K, Kuhn J, Prescott C. Childhood sexual abuse, stressful life events and risk for mayor depression in women. Psychol Med 2004; 36: 147-82.

22. Ducrocq F, Vaiava G, Cottencini O, Molenda S, Bailly D. Post-traumatic stress, post-traumatic depression and major depressive episode: literature. Encephale 2001; 27 (2): $159-68$

23. Vicente B, Rioseco P, Saldivia S, Kohn R, Torres S. Estudio chileno de prevalencia de patología psiquiátrica (DSM-III-R/CIDI) (ECPP). Rev Med Chile 2002; 130: 527-36.

24. Protocolo de Tratamiento Personas con Depresión Severa. Nivel Secundario de Atención. Ministerio de Salud Chile. Retamal P, Coordinador, 2004.

25. Guía Clínica para el Tratamiento de Personas con Depresión. Ministerio de Salud Chile, Unidad Salud Mental, 2006.

26. Guía Clínica para el Tratamiento de Personas con Depresión. Ministerio de Salud Chile, Unidad Salud Mental, 2009.

27. Kirsch TD, Mitrani-Reiser J, Bissell R, Sauer LM, Mahoney $\mathrm{M}$, Holmes WT, et al. Impact on hospital functions following the 2010 Chilean earthquake. Disaster Med Public Health Prep 2010; 4 (2): 122-8.

28. Ballesteros S, Vitriol V, Florenzano R, Calderón A, Vacarezza A. Mujeres con depresión severa: Relación entre trauma infantil y gravedad de síntomas clínicos. Rev Chil Neuro-Psiquiatría 2007; 45: 288-95.

29. Vitriol V, Ballesteros S, Florenzano R, Weil K, Benadof D. Evaluation of an outpatient intervention for women with severe depression and a history of childhood trauma. Psychatr. Serv. 2009; 60: 636-942.

30. Vitriol V, Cancino A, Florenzano R, Ballesteros S, Schwartz D. Eficacia y costos asociados a un tratamiento ambulatorio en mujeres con depresión severa y trauma temprano. Rev Med Chile 2010; 138: 428-36.

31. Vitriol V, Cancino A, Ballesteros S. Mujeres con depresión y trauma relacional temprano: una propuesta de intervención operacionalizada en la red pública de salud. Rev GPU 2010; 6 (4): 501-10.

32. CIE-10, Clasificación Internacional de Enfermedades, décima edición, (OMS) Descripciones Clínicas y pautas para el Diagnóstico. Madrid: Ediciones Meditor, 1992.

33. Japanesse Society of Psychiatry and Neurology. Great East Japan Earthquake and early mental health response. Psychiatry and Clinical Neurosciences 2011; 65: 539-48.

34. Figueroa RA, Marín H, González M. Apoyo psicológico en desastres: Propuesta de un modelo de atención basado en revisiones sistemáticas y meta-análisis. Rev Med Chile 2010; 138: 143-51.

35. World Health Organization. Mental health in emergencies. Geneva 2003.

36. Staab JP, Guiguer TA, Fullerton CS, Ursano RJ. Acute stress disorder, subsequent posttraumatic stress disorder and depression after a series of typhoons. Anxiety 1996; 2 (5): 219-25.

37. Cahill S, Pontoski K. Post-traumatic stress disorder and acute disorder. Psychiatry 2005; 2: 14-25.

38. Shalev A. Posttraumatic stress disorder (PTSD) and stress related disorders. Psychiat Clinics North Am 2009; 32 (3): 687-704.

39. Vitriol V, Ballesteros S, Florenzano R, Calderón A, Vacarezza A, Schwartz D. Relación entre trauma infantil y comorbilidad de depresión con trastorno de estrés postraumático Rev Trastor. Animo 2008; 2: 101-8.

40. Cloitre M. Effective psychotherapies for posttraumatic stress disorders: review and critique. CNS Spectr 2009; 14: 1 (1): 32-43. 